\title{
Tangence
}

\section{Le " décentrement " de l'Acadie : la poésie de Serge Patrice Thibodeau}

Jean Morency

Numéro 58, octobre 1998

Le postmoderne acadien

URI : https://id.erudit.org/iderudit/025979ar

DOI : https://doi.org/10.7202/025979ar

Aller au sommaire du numéro

Éditeur(s)

Tangence

ISSN

0226-9554 (imprimé)

1710-0305 (numérique)

Découvrir la revue

Citer cet article

Morency, J. (1998). Le « décentrement » de l'Acadie : la poésie de Serge Patrice Thibodeau. Tangence, (58), 47-55. https://doi.org/10.7202/025979ar d'utilisation que vous pouvez consulter en ligne.

https://apropos.erudit.org/fr/usagers/politique-dutilisation/ 


\section{Le «décentrement » de l'Acadie: la poésie de Serge Patrice Thibodeau Jean Morency}

Dans la production littéraire de l'Acadie d'aujourd'hui, l'œuvre de Serge Patrice Thibodeau fait un peu cavalier seul. Le décentrement est au coeur de l'élaboration et de l'écriture de cette œuvre (ou de cet œuvre, puisqu'il faut peut-être utiliser ici le masculin, du moins dans l'optique de l'auteur, qui semble percevoir ainsi son entreprise esthétique): natif de Rivière-Verte, dans le comté de Madawaska, Thibodeau ne fait pas partie des écrivains qui ont choisi Moncton comme lieu d'écriture, lui préférant la ville de Montréal, quand ce n'est pas Prague, Vienne, Budapest ou Paris. À première vue, il n'y a rien d'acadien dans l'univers imaginaire des principaux recueils de Thibodeau (La septième chute, Le cycle de Prague, Le quatuor de l'errance), et pourtant l'Acadie y est partout, mais elle s'y trouve comme décentrée, profondément intégrée dans la conscience du poète et dans le regard qu'il porte sur l'ailleurs. Au fond, la problématique "nationale" que posent les livres de Thibodeau ne va pas sans évoquer celle qui a alimenté, au Québec, la querelle entre les régionalistes et les exotistes, à la différence près que Thibodeau va en arriver à inscrire sa propre errance et sa quête spirituelle dans celle du peuple acadien, qui la surdétermine et lui donne tout son sens, comme on le verra dans les pages qui suivent.

Reprenant une expression de François Ricard, Janet Paterson écrit, dans Moments postmodernes dans le roman québécois: "Les dieux ont chaviré: voilà une autre façon cle décrire la condition postmoderne." Même si l'œuvre de Serge Patrice Thibodeau semble échapper à toute tentative de catégorisation, force est d'admettre que la sensibilité qui la traverse s'inscrit dans cette condition postmoderne: une certaine méfiance à l'égard de la collectivité, jointe à l'expression d'un sentiment mystico-religieux, notamment dans le quatuor de l'errance, en font une ouvre de

1 Janet M. Paterson, Moments postmodernes dans le roman québécois, édition augmentée, Ottawa, Les Presses de l'Université d'Ottawa, 1993, p. 17. 
48

rupture, assez insolite dans le paysage acadien actuel. Les dieux ont chaviré, certes, mais ils sont toujours là, attendant patiemment dans l'ombre, dans l'envers de la représentation. Dans la poésie de Serge Patrice Thibodeau, la condition minoritaire et l'identité nationale se sont estompées pour laisser place à une conscience beaucoup plus individuelle, mais sans cesser pour autant de manifester leur présence, mais sur un autre mode, selon de nouvelles tonalités. La paratopie de l'écrivain, telle que la définit Dominique Maingueneau, semble jouer à plein dans la poésie de Thibodeau et rendre bien compte de la position particulière de cet écrivain dans le champ littéraire acadien. Selon Maingueneau, "[l]oin d'énoncer sur un sol institutionnel neutre et stable, l'écrivain nourrit son ouvre du caractère radicalement problématique de sa propre appartenance au champ littéraire et à la société " 2 . Ce caractère problématique rejaillit partout dans l'œuvre de Thibodeau, notamment dans la prise de conscience de la solitude du poète face à la "tribu" littéraire (le terme est de Maingueneau) et dans la mise en place des conditions d'énonciation de son écriture poétique (Montréal, Vienne, Prague, Jérusalem, etc.). Mais cet écartèlement va s'avérer justement ce qui définit l'appartenance de l'individu au groupe acadien, l'uacadianité. la plus radicale étant celle qui se rapporte à l'expérience qui est faite de l'arrachement, de l'exil et de l'étrangeté. Maingueneau écrit encore que l'appartenance au champ littéraire est "une localisation parasitaire, qui vit de l'impossibilité même de se stabiliser" ${ }^{3}$.

Dans cette perspective, le poète acadien est un écrivain dont le statut se trouve surdéterminé par sa propre appartenance collective, fondée justement sur le sentiment d'une marginalité qui lui est constitutive. Cette impression sera d'autant plus forte que le poète sera exilé. Voici ce que qu'on pourrait appeler le paradoxe de l'écrivain acadien: la rupture avec la collectivité, vécue au moyen de l'écriture et de l'exil, s'avère pourtant le chemin le plus court vers la collectivité ${ }^{4}$. On peut faire référence, une fois de plus, au propos de Maingueneau:

2 Dominique Maingueneau, Le contexte de l'cuvre littéraire. Énonciation, écrivain, société, Paris, Dunod, 1993, p. 27.

3 Ibid., p. 28.

4 C'est d'ailleurs en ce sens que l'on peut interpréter le titre d'un recueil de Thibodeau paru en 1995, Nous, l'étranger, oxymoron exprimant de manière admirable le paradoxe dont il est ici question. 
La situation paratopique de l'écrivain l'amène à s'identifier à tous ceux qui semblent échapper aux lignes de partage de la société : bohémiens, mais aussi Juifs, femmes, clowns, aventuriers, Indiens d'Amérique..., selon les circonstances. Il suffit que la société se crée une structure paratopique pour que la création littéraire puisse être attirée dans son orbite. ${ }^{5}$

La structure paratopique acadienne s'inscrit ainsi parfaitement dans le projet esthétique de Thibodeau; je ne veux pas dire qu'elle conditionne ce projet, mais qu'elle en vient à le légitimer en le singularisant. C'est l'errance personnelle du poète qui appelle l'errance collective, et non l'inverse. Thibodeau trouve ainsi dans l'errance acadienne le modèle paradigmatique de sa propre odyssée.

On peut en prendre à témoin le deuxième des trois recueils qui composent la septième chute, intitulé emphatiquement "oser rincer les gloses d'eaux bues", qui regroupe des poèmes dédiés à des fleuves (la Vistule, la Vltava, le Danube, la Seine... et, en post-scriptum, le Saint-Laurent) ${ }^{6}$. Derrière l'exotisme apparent de cette poésie de l'exil et de l'étrangeté, qui tente vainement de se fixer dans l'écoulement triste de l'existence des villes d'Europe centrale, se dessine une subjectivité toujours proche du contexte acadien. L'expérience de l'exil dans des villes étrangères ne correspond jamais à une dissolution de l'être dans l'anonymat et l'inexistence (comme on pourrait $s^{\prime} y$ attendre), mais permet justement au poète d'accéder pleinement à l'existence. On pourrait ainsi concevoir que pour l'écrivain de condition minoritaire, l'expérience de sa propre altérité dans un ailleurs, et à plus forte raison si cet ailleurs s'avère d'une radicale étrangeté, prend une forme positive. D'une part, cette expérience permet d'échapper au "nous" collectif tout en y revenant sans cesse, de se sentir autre, non plus parmi les siens, mais parmi les autres, donc de saisir enfin sa propre identité individuelle, dont la prise de conscience ramène justement à l'appartenance collective. D'autre part, elle vient gommer le rapport complexe avec l'autre tel qu'il est vécu dans le contexte canadien, d'échapper au regard débilitant de l'autre qui ne cesse de confronter le minoritaire à la conscience de sa marginalité, voire de son invisibilité.

5 Dominique Maingueneau, op. cit., p. 36.

6 L'image du fleuve, c'est-à-dire d'une identité mouvante mais pérenne, constitue d'ailleurs une donnée essentielle de la poésie de Thibodeau. 
50

Dans ces villes d'Europe centrale qui sont dotées d'un fort coefficient d'étrangeté (surtout jusqu'à la chute du Mur de Berlin en 1989), le rapport avec l'autre est radicalement différent. De l'autre côté du miroir, ce sont tout à coup les autres qui prennent des allures bizarres, fantomatiques, et qui vous font accéder à l'existence. Certes, à Varsovie, sur les rives de la Vistule, le poète s'interroge sur son altérité, mais s'il le fait c'est pour mieux se saisir : " mais pourquoi venir si loin pour être un peu/ autre/ [...]/ se questionner, ainsi prouver sa propre existence?» 7 Mais à Prague, dans cette ville emblématique de l'étrangeté, devant le tombeau de Kafka (qui s'y connaissait en la matière), c'est surtout la rencontre de l'individu et de la collectivité qui est mise en évidence; l'Acadie n'est au fond nulle part aussi présente qu'au "contact des vieilles pierres" 8 et de leur mémoire douloureuse: "nous plaindre: voués aux grands déplacements,/ aux perpétuelles déportations, à l'exil/ à l'errance. "9 À Vienne, cette conscience de la déportation est toujours présente, quoique profondément intériorisée et intégrée au cheminement de l'individu: "l'exil/ comme remède/ à lanachronisme?/ assumer/ sa déportation/ ouvre grand les portes. " ${ }^{10}$ Au fond, et ce point est révélateur, il n'y a qu'à Paris que l'expérience de l'altérité est vécue sur le mode de l'inconfort: l'impression d'étrangeté n'y est sans doute pas assez prononcée, et ne fait que renvoyer à la conscience douloureuse de la minorisation. Situation symptomatique de la condition minoritaire en effet que cette difficulté de vivre au coeur même de la culture majeure. L'étranger a désespérément besoin de trouver une étrangeté plus grande que la sienne pour atteindre pleinement à l'existence: "n'est pas à mon gré/ la ville dont la lumière/ n'est pas la mienne./ ville-poussière./ à ma portée, davantage là/ ces hommes basanés/ qu'elle méprise. " ${ }^{11}$

On peut donc constater que Le cycle de Prague et Le quatuor de l'errance, les deux recueils de Thibodeau qui ont connu le plus grand retentissement, étaient déjà en germe dans La septième chute. Le décor pragois aura ainsi influencé énormément le poète acadien, et on peut se poser légitimement la question: pourquoi

7 Serge Patrice Thibodeau, La septième chute. Poésie 1982-1989, Moncton, Éditions d'Acadie, 1990, p. 66.

8 Ibid., p. 73.

9 Ibid., p. 74 .

$10 \quad$ Ibid., p. 107.

11 Ibid., p. 97. 
Prague? Dans sa préface au Cycle de Prague, Thibodeau écrit qu'il ne saurait tenter qu'une seule réponse à cette question, et encore le fait-il sous une forme lyrique: "Éprouver des sentiments pour une ville:/ se tenir debout, les yeux couverts d'une couche de cendres,/ et dans la main crispée,/ tenir serrée l'extrémité rougie d'un tisonnier./ La nuit, le geste précède le silence./ Ô parcours./ Le besoin de partir crépite à l'horizon." 12 Il y aurait ainsi, au fondement de l'expérience poétique, une expérience de la voyance, un regard intérieur qui supplée à la vision commune et qui permet de parcourir l'espace pragois, rendu pareil au lieu aveugle de la mémoire avec les courbes de son fleuve, symbole de l'écoulement temporel, et la magie de son architecture baroque, qui fournit l'image d'une résistance au passage du temps. Ce serait d'ailleurs pourquoi la première partie du recueil, "Orants et nus dans la pénombre", est construite autour de certaines figures littéraires et artistiques qui servent littéralement de prétextes aux poèmes: d'Anna Akhmatova à Marguerite Yourcenar, en passant par James Baldwin, René Char, l'incontournable Franz Kafka, Boris Pasternak, etc., Thibodeau fait voyager le lecteur à travers son abécédaire personnel (on notera en effet que les figures sont sollicitées selon une progression alphabétique), donc à travers sa propre mémoire. L'expérience de l'exil débouche ainsi sur l'établissement d'une nouvelle communauté, spirituelle celle-là, et qui se rattache elle aussi à la condition paratopique de l'écrivain: "tout écrivain s'incrit dans une tribu d'élection, celle des écrivains passés ou contemporains, connus personnellement ou non, qu'il place dans son panthéon personnel et dont le mode de vie et les ouvres lui permettent de légitimer sa propre énonciation. "13 Chez Thibodeau, cette légitimation de l'énonciation constitue une première réaction au décentrement et à la solitude qui lui est rattachée. Car Prague, c'est aussi la métonymie de l'ailleurs, de n'importe quel ailleurs, et par conséquent le lieu de l'exil total, qui permet le face-à-face entre l'être et l'étranger qui l'entoure et l'habite à la fois. D'une certaine façon, Prague est l'aboutissement logique de l'errance acadienne, le seul terme possible de la déportation, l'équivalent terrestre de la Jérusalem céleste qui marque l'aboutissement du Quatuor de l'errance suivi de La traversée du désert.

12 Serge Patrice Thibodeau, Le cycle de Prague. Poésie, Moncton, Éditions d'Acadie, 1992, p. 9. C'est l'auteur qui utilise les caractères gras.

13 Dominique Maingueneau, op. cit., p. 31. 
52

Cet aboutissement devait néanmoins passer par une reconnaissance de l'espace nord-américain, qui prend place dans $L e$ passage des glaces et surtout dans Nous, l'étranger. Le lieu d'énonciation du premier recueil se situe à Montréal, espace moins exotique que Prague mais encore plus marqué du sceau de l'exil et de l'étrangeté. Un sentiment d'enfermement et de dépossession habite en effet Le passage des glaces, qui comporte deux épigraphes, l'une de Franz Kafka ("Avoir le sentiment pénible d'être attacbé et sentir en même temps que si l'on vous détachait, ce serait encore plus terrible"), l'autre de René Char ("La rigueur de vivre se rode sans cesse à convoiter l'exil"), qui en définissent le programme de lecture. Le Montréal du Passage des glaces ne va pas sans évoquer le Paris de La septième chute: même espace hostile, perçu au mieux comme un lieu de passage, dans l'attente du départ et du recommencement de l'errance. Il y aurait ainsi, dans la poésie de Thibodeau, une impossibilité de vivre dans un contexte majoritaire, ce contexte exerçant une action débilitante sur une identité justement associée à la minorisation et à la coexistence avec l'autre, et une autre impossibilité, celle de l'exil sans retour: "Ces jours déplacés:/ la potence de la durée./ nous, intempestifs et déplacés,/ mal placés./ enfin. toujours l'exil./ toujours la cruelle bourrasque de l'exil. " 14

Ce dilemme est au fondement de Nous, l'étranger, tentative de réintégration et de déchiffrement de l'errance acadienne, depuis le départ de La Rochelle en 1654 (première partie du recueil) jusqu'à l'installation à Chipoudie, 1697-1755 (deuxième partie) et finalement à l'établissement au Madawaska en 1789 (troisième partie). Nous, l'étranger semble donc marquer un virage important dans l'œuvre de Thibodeau. L'Acadie occupe maintenant l'espace énonciatif, même si le "nous" et "l'étranger" restent en position dialogique. Encore une fois, c'est la situation paratopique de l'écrivain qui est mise en cause:

Beaucoup d'écrivains, déniant le "tribalisme" littéraire, voire le champ littéraire, prétendent ne relever que d'eux-mêmes. Mais on a beau se retirer au désert, dans les forêts ou les montagnes, on ne peut sortir du champ littéraire dès lors qu'on écrit, publie et que lon organise son identité autour de cette activité. ${ }^{15}$

14 Serge Patrice Thibodeau, Le passage des glaces, Trois-Rivières et Moncton, Écrits des Forges et Perce-Neige, 1992, p. 44.

15 Dominique Maingueneau, op. cit., p. 31. 
Le champ littéraire acadien vient donc investir l'écriture de Thibodeau, comme en témoignent d'ailleurs les épigraphes de Raymond Guy LeBlanc, de Ronald Després et de Gérald Leblanc qui coiffent les trois parties composant le recueil et qui contribuent à l'émergence de son sens. Notons que ce ne ne sont plus les auteurs consacrés de la littérature universelle qui sont sollicités, mais des écrivains de la modernité acadienne. L'épigraphe de Raymond Guy LeBlanc appelle à une suite du monde, à un prolongement de son Cri de terre dans le sens d'une plus grande ouverture au monde et à la poésie: "j'inventerai le monde/ et vous viendrez pour lui donner/ toute sa densité d'humaine poésien ${ }^{16}$. Celle de Ronald Després légitime quant à elle la quête littéraire et sprirituelle de Thibodeau: "Tu es, mon Acadie/ - Et sans douleur cette fois - / Pays de partance. ${ }^{17}$ Quant à celle empruntée à Gérald Leblanc, elle renvoie à cette Acadie multiple et ouverte qui peuple les poèmes de l'auteur de Rivière-Verte: "je comprends que toutes les Acadie que j'ai connues/ reviennent et renvoient/ jusqu'au bout du monde appris sur un chemin de terre." ${ }^{18}$ Dans une certaine mesure, Nous, l'étranger apparaît comme une œuvre métalittéraire constituant le prolongement du discours acadien contemporain : "ces livres, me sera-t-il donné un jour d'en imiter/ la transparence et le rayonnement "19, écrit d'entrée de jeu le poète. L'Acadie traversée au cours de ce voyage dans l'espace et surtout dans le temps est avant toute chose celle des mots et de la langue: "parallèles au voyage s'ouvrent les livres/ enluminés d'arabesques et de marges fleuries" 20 .

On comprendra néanmoins que le décentrement de l'Acadie reste au cœur de l'entreprise poétique de Thibodeau. La prise en charge de l'altérité par le "nous" (devant nous qui savons que nous sommes l'étranger" ${ }^{21}$ ) contribue à arracher la parole et l'identité acadiennes à un espace physique perçu comme illusoire, pour plutôt situer celles-ci dans le mouvement sans fin qui les caractérise depuis le départ des premiers émigrants de La

16 Serge Patrice Thibodeau, Nous, l'étranger, Trois-Rivières et Echternach (Luxembourg), Écrits des Forges et Éditions Phi,1995, p. 8.

17 Ibid., p. 58.

18 Ibid., p. 72.

19 Ibid., p. 9.

20 Ibid., p. 51.

21 Ibid., p. 47. 
54

Rochelle. Comment pourrait-on vouloir se fixer quand c'est le mouvement lui-même qui détermine l'identité collective? L'errance se trouve du même coup valorisée ou à tout le moins engagée dans un processus euphémisant. On pourrait mentionner ici l'œuvre de Saint-Denys Garneau, à laquelle Thibodeau a déjà consacré un essai ${ }^{22}$, pour saisir cette notion d'une identité où le repos ne réside jamais dans la fixité mais dans un équilibre tout aérien dans l'espace ("C'est là sans appui que je me repose"). Il existe ainsi une parenté évidente entre l'imaginaire de Thibodeau, qui ne craint rien tant que d'être "ensablé dans les meubles" ${ }^{23}$, et celui de Garneau ("Je ne suis pas bien du tout assis sur cette chaise"). Voici l'Acadie de nouveau décentrée, arrachée de ses frontières, mais dans un sens positif cette fois: "se départir de la terre glacée sous les pieds/ revendiquer pour soi seul la chamure de l'odyssée/ et se repaître de sa chaleur "24.

En conclusion, il serait aussi important de noter que cette " déspatialisation " de l'identité acadienne correspond à son inscription dans le temps, et par conséquent dans la culture universelle. Ce refus du confinement dans l'espace contribue en effet à la légitimation de l'acte énonciatif dans la poésie de Thibodeau. Dans Les littératures de l'exiguïté, François Paré s'est penché sur la dialectique du temps et de l'espace qui distingue les littératures majeures des autres:

Si les petits peuples n'ont pas d'histoire, comme on dit, les grandes cultures, elles, sont dépourvues d'espace, c'est-à-dire qu'elles s'instituent à même des mécanismes de "déspatialisation". Elles ont depuis longtemps fui les terres sacrées. À ce titre, ce sont les cultures nationales de l'Europe occidentale qui ont le mieux réussi, clepuis la Renaissance sans doute, à évacuer leur espace spécifique. On voudrait ainsi que la collectivité nationale soit issue de partout et de nulle part; toute l'institution culturelle européenne a conçu sa grandeur comme une déréalisation de son espace spécifique. En Italie, en France et puis partout en Europe, la redécouverte de l'Antiquité classique à partir du xve siècle confirmait le modèle de "déspatialisation" de la culture qui allait éventuellement clépartager les discours dominants, sanctionnés par les cultures antiques, et les autres,

22 Serge Patrice Thibodeau, L'appel des mots. Lecture de Saint-Denys-Garnean, Montréal, Éditions de l'Hexagone, 1993.

23 Nous, l'êtranger, op. cit., p. 49.

24 Ibid. 
condamnés à l'insignifiance historique (spatialisées à outrance, les cultures dominées échappent à l'Histoire/Temps) ${ }^{25}$.

Dans une certaine mesure, la poésie de Serge Patrice Thibodeau cherche à provoquer ce mouvement, non pas dans une perspective institutionnelle certes, mais en conformité avec sa volonté de légitimer sa propre énonciation. Comme on l'a noté, les références qui sont faites, dans toute son ouvre, à des écrivains pour la plupart étrangers, participent du même mouvement clésespéré de s'arracher à l'Acadie et d'arracher l'Acadie à elle-même, c'est-àdire à un espace perçu comme aliénant. Cela dit, on peut observer, dans ce geste même, une conception somme toute très académique de la culture et de la littérature, ce qui pourrait expliquer le malaise que certains ne manquent pas de ressentir à l'égard de l'œuvre de Thibodeau, œuvre trop volontairement écrite, peut-être, pour correspondre vraiment à la sensibilité de son temps.

25 François Paré, Les littératures de l'exiguitté, Hearst, Le Nordir, 1992, p. 70-71. 